\title{
Rho protein crosstalk: another social network?
}

\author{
Christophe Guilluy, Rafael Garcia-Mata, and Keith Burridge \\ The University of North Carolina at Chapel Hill, Chapel Hill, NC, USA
}

\begin{abstract}
Many fundamental processes in cell biology are regulated by Rho GTPases, including cell adhesion, migration and differentiation. While regulating cellular functions, members of the Rho protein family cooperate or antagonize each other. The resulting molecular network exhibits many levels of interaction dynamically regulated in time and space. In the first part of this review, we describe the main mechanisms of this crosstalk, which can occur at three different levels of the pathway: (1) through regulation of activity, (2) through regulation of protein expression and stability, and (3) through regulation of downstream signaling pathways. In the second part, we illustrate the importance of Rho protein crosstalk with two examples: integrin-based adhesion and cell migration.
\end{abstract}

\section{Connecting Rho family members}

All eukaryotic cells contain Rho GTPases (ranging from 6 in yeast to $\sim 20$ in mammals) and they are implicated in the regulation of many biological processes, from adhesion and motility to gene expression and differentiation ${ }^{1}$. As a consequence of their biological ubiquity, Rho proteins often cooperate or antagonize each other to control cellular tasks. This interaction between Rho family members relies on a complex molecular dialogue occurring at different levels in their signaling pathways. The first observation of an interaction between two Rho proteins was made by Ridley et al. in 1992. In their seminal paper, they showed that ruffle formation in growth factor-stimulated fibroblasts was due to Rac1 and that this led to stress fiber formation in a RhoA-dependent manner ${ }^{2}$. Since then, an amazing variety of mechanisms have been described that interconnect the members of the Rho family.

Cycling between an inactive GDP state and an active GTP state, Rho proteins are usually compared to molecular switches. Three classes of proteins regulate their cycle: guaninenucleotide exchange factors (GEFs), GTPase activating proteins (GAPs) and guanine nucleotide dissociation inhibitors (GDIs) ${ }^{1}$. GEFs activate Rho proteins by catalyzing the exchange of GDP for GTP ${ }^{3}$, whereas GAPs stimulate the intrinsic GTPase activity and promote the return to the inactive state 4 . The inactive pool of Rho proteins is maintained in the cytosol by association with GDI. In the active GTP-bound conformation they interact with effectors and perform their functions. The reader is directed to recent comprehensive reviews for information about Rho protein regulation, Rho GEFs, GAPs, GDI and effectors ${ }^{1,3-6}$. Here, we will focus on the pathways and proteins that connect Rho proteins with each other. After discussing several specific mechanisms, we will illustrate the

\footnotetext{
(c) 2011 Elsevier Ltd. All rights reserved.

Publisher's Disclaimer: This is a PDF file of an unedited manuscript that has been accepted for publication. As a service to our customers we are providing this early version of the manuscript. The manuscript will undergo copyediting, typesetting, and review of the resulting proof before it is published in its final citable form. Please note that during the production process errors may be discovered which could affect the content, and all legal disclaimers that apply to the journal pertain.
} 
importance of these interactions with two examples, integrin-based cell adhesion and cell migration, in which coordination between Rho proteins is essential.

\section{Molecular mechanisms of Rho protein crosstalk}

Different modes of interaction between Rho GTPases are illustrated in Figure 1. There are three main levels at which Rho family members interact: (i) regulation of activity (i.e. via a GEF or a GAP); (ii) regulation of protein expression and stability, in which RhoGDI is important; and (iii) regulation of downstream signaling pathways.

\section{Crosstalk via GEFs and GAPs}

The quintessential interaction between Rho proteins is illustrated by RhoA and Rac1, two ubiquitous and well studied family members. Selective activation of one Rho protein is easily achieved when a signaling pathway acts on a GEF with a single specificity. However, many GEFs (e.g. Vav2) can activate multiple Rho proteins, including both RhoA and Rac1. There may be pathways where both proteins are simultaneously activated, but in many situations the activation of RhoA and Rac1 appears to be separated either temporally or spatially, or one of the proteins is activated and the other inhibited. There are several examples where RhoA and Rac1 modulate each other through regulation of GEFs and GAPs (Figure 2 and Table 1). Although Rac1 was originally identified as stimulating RhoA activity, in most situations these two proteins exhibit an antagonistic relationship that operates at multiple levels. This opposition can be reciprocal or unidirectional, as was observed in a classic study in which Rac1 activation in NIH3T3 cells induced an epithelial morphology, including cadherin-based junctions and was accompanied by decreased RhoA activity ${ }^{7}$. Elevated RhoA activity reversed the phenotype promoting a mesenchymal fibroblastic morphology but did not inhibit Rac1 activity?

\section{RhoA inhibition of Rac1}

Inhibiting the RhoA effector Rho-associated kinase (ROCK) induces membrane protrusions at random positions around a cell's periphery and this was observed to be due to increased Rac1 activity 8 , 9 . This led to the idea that ROCK is involved in the suppression of Rac1 activity by RhoA. Pursuing the mechanism for this revealed that ROCK can phosphorylate and activate FilGAP, a Rac-specific GAP ${ }^{10}$. The authors of this work showed that depletion of FilGAP significantly reduced ROCK-dependent Rac1 inactivation. Similarly, a member of the same subfamily of GAPs, ArhGAP22 mediates RhoA-dependent Rac1 inhibition in melanoma cells ${ }^{11}$. ROCK is also responsible for RhoA-dependent ArhGAP22 activation; however, in this case the mechanism may not involve direct phosphorylation of the GAP by ROCK because GAP activation is inhibited by blocking cellular contractility with the myosin II inhibitor blebbistatin, suggesting a more complex mechanism ${ }^{11}$. Another way that mechanical tension can decrease Rac1 activity was suggested by proteomic analysis comparing adhesions under conditions where myosin activity was or was not inhibited. This study showed that the GEF $\beta$-Pix was responsible for Rac1 activation in nascent integrin adhesions and that actomyosin contractility induced $\beta$-Pix dissociation from these adhesions ${ }^{12}$. Conversely, ROCK inhibition induced recruitment of $\beta$-Pix to the adhesion, indicating that ROCK inhibits Rac1 within the adhesion at least in part by regulating $\beta$-Pix localization. In a parallel study, $\beta$-Pix and another Rac1 GEF, DOCK180, were seen to be displaced from the large stable adhesions that form at the rear of cells in association with stabilized actomyosin filament bundles ${ }^{13}$.

\section{Rac1 inhibition of RhoA}

Mirroring RhoA-dependent Rac inhibition, Rac1 can also control RhoA activity ${ }^{7}$. Active Rac1 binds and activates p190RhoGAP (isoform B) providing a direct mechanism by which 
active Rac1 can depress RhoA activity ${ }^{14}$. Interestingly, Rac1 controls p190RhoGAP activity through another mechanism; Rac1-mediated production of reactive oxygen species (ROS) inhibits a tyrosine phosphatase (Low molecular weight protein tyrosine phosphatase), leading to an increase in p190RhoGAP tyrosine phosphorylation and catalytic activity ${ }^{15}$. The effects of ROS on Rho protein activity are complex, since other work showed that direct ROS-mediated oxidation of a cysteine in RhoA leads to RhoA activation rather than inhibition ${ }^{16}$. This latter pathway may occur in situations where there is a positive stimulation of RhoA activity downstream of Rac1.

Just as the RhoA effector ROCK mediates some of the downregulation of Rac1 activity, so too the Rac/Cdc42 effector p21 associated kinase (PAK) contributes to Rac1's suppression of RhoA signaling. Notably, PAK regulates the activities of multiple RhoA-specific GEFs. PAK1 phosphorylates p115-RhoGEF thereby inhibiting its catalytic activity ${ }^{17}$. Interestingly, this study showed that inhibition of PAK1 significantly increased RhoA activation in response to thrombin, showing that PAK1 may inhibit RhoA activity in physiological contexts; however, this may not involve exclusively p115-RhoGEF down regulation. PAK1 can also phosphorylate the RhoA GEF Net1 on three serine residues ${ }^{18}$ and Net1 phosphomimetic mutants have less nucleotide exchange activity toward RhoA in vitro and in vivo. Another GEF, PDZ-RhoGEF is phosphorylated and inhibited by PAK $4^{19}$, but PAK4 belongs to the group II PAK proteins which have biochemical properties dissimilar to the group I PAKs. PAK4 binds to active Cdc42 and to a lesser extent to active Rac, and binding these GTPases only moderately enhances PAK4 kinase activity ${ }^{20}$. This suggests that in vivo PAK4 may inhibit PDZ-RhoGEF independently of Rac1. GEF-H1 (Lfc), another RhoA GEF, is a substrate for both PAK1 and PAK4, and GEF-H1 phosphorylation is associated with decreased RhoA activity, loss of stress fibers and increased lamellipodia, consistent with increased Rac1 activity ${ }^{21,22}$.

\section{Positive feedback between RhoA and Rac1}

Although the majority of the mechanisms connecting RhoA and Rac1 lead to mutual inhibition, some studies, including the initial work from Ridley et al. ${ }^{2}$, have shown that they can also activate each other. Rac1-GTP binds the PH domain of Dbs, a RhoA GEF 23, 24, and stimulates its catalytic activity, leading to RhoA activation. This mechanism seems to be cell-type specific because in breast cancer cells expression of Dbs leads not only to RhoA and Cdc42 activation, but also to Rac1 activation through an unknown indirect mechanism ${ }^{25}$. In contrast to ROCK, mDia (mouse Diaphanous related formin) seems to stimulate Rac1 activity. By comparing the effect of the $\mathrm{C} 3$ exoenzyme and the ROCK inhibitor on 3T3 fibroblasts, it was found that ROCK inhibition induced Rac1-dependent protrusions, whereas treatment with the $\mathrm{C} 3$ exoenzyme $\operatorname{did~not}^{9}$. Moreover, combined ROCK inhibition and expression of a dominant negative of mDia1 prevented protrusion formation, suggesting that mDia1 positively regulates Rac1 activity. The exact molecular mechanism linking mDia1 to Rac1 is unknown and does not necessarily involve regulation of a Rac GEF or GAP.

\section{Other Rho proteins that affect Rac1 and RhoA activities}

RhoG, which belongs to the Rac subfamily of Rho GTPases, was initially suggested to function by controlling Rac1 activity ${ }^{26}$. ELMO was identified as an effector for $\mathrm{RhoG}^{27}$ and shown to form a complex with Dock180, a Rac-specific GEF ${ }^{28,29}$. In vivo, the interaction between Dock180 and ELMO is necessary to efficiently stimulate nucleotide exchange activity on Rac1 ${ }^{28}$. The interaction of RhoG with ELMO induces translocation of the ELMO-Dock180 complex from the cytoplasm to the plasma membrane and activates Rac $1^{27}$. Thereby, many of the effects of RhoG are due to its downstream activation of 
Rac $1^{30}$. However, RhoG can also signal independently or act in parallel, sharing several effectors with Rac1 $1^{31-33}$.

The Rnd subgroup of Rho proteins, which are only found in vertebrates, are especially interesting in the context of crosstalk since they appear to signal predominantly through the inhibition of RhoA and RhoA-mediated contractility ${ }^{34}$. Unlike other Rho proteins, members of the Rnd subgroup are always bound to GTP and are not controlled by GEFs or GAPs. Rather, the Rnd proteins appear to be regulated at the transcriptional level ${ }^{34}$. In terms of their inhibition of the RhoA signaling pathway, two mechanisms have been identified. One study demonstrated that Rnd 1 and Rnd3 bind and activate p190RhoGAP leading to decreased RhoA activity ${ }^{35}$. Rnd3 (RhoE) was independently shown to bind and inhibit the RhoA effector, ROCK $1^{36}$. In an interesting example of negative feedback, Rnd3 was found to be a substrate for ROCK1 and phosphorylation was shown to enhance Rnd3 stability and promote ROCK1 inhibition ${ }^{37}$.

As mentioned above, there are numerous examples where different Rho proteins can be substrates for the same GEF or $\mathrm{GAP}^{3,4}$. Additionally, some proteins contain multiple domains regulating different GTPases. For example, the Trio family of Rho GEFs contain two GEF domains with distinct specificities, one for RhoA and one for Rac and RhoG ${ }^{38}$. Abr and Bcr both possess a GAP domain, specific for Rac1 and Cdc42, and a GEF domain, specific for RhoA, Rac1 and Cdc42 ${ }^{39}$. Recently, it was shown that Abr regulates local RhoA activation and Cdc42 inactivation during the wound healing response in Xenopus oocytes $^{40}$. During the closure of a small wound in an oocyte, a zone of active RhoA surrounds the wound that is, in turn, encircled by a region of active Cdc42. Abr is responsible for the zone of active RhoA and for inactivating Cdc 42 within this region ${ }^{40}$. With regulators that have multiple catalytic domains, it would be interesting to know if the activity of one influences the activity of the other. For example, does Cdc42 binding to the GAP domain of Abr or Bcr affect the activity of the GEF domain specific for RhoA?

\section{Crosstalk via GDI}

Compared to GEFs and GAPs, the family of RhoGDI proteins acts very differently on Rho GTPases $^{6}$ (Figure 1). Whereas the number of GEFs and GAPs greatly outnumber the GTPases, there are only three conventional GDIs, immediately implying that many GTPases can bind to a single GDI. RhoGDI1 (RhoGDI $\alpha$ ) is ubiquitous and is the most studied. It appears to bind most Rho GTPases, although its interaction with RhoB differs in different studies and may reflect that RhoB can be palmitoylated close to its $C$ terminus and this palmitoylation would likely block binding to RhoGDI ${ }^{41}$. RhoGDI2 (RhoGDI $\beta$, Ly-GDI, D4GDI) is mainly found in hematopoietic tissues, but is also expressed in numerous tumors. RhoGDI3 (RhoGDI $\gamma$ ) is expressed at low levels in many tissues but particularly in the brain, lungs and testes. RhoGDI1 and RhoGDI2 are cytosolic proteins but RhoGDI3 contains an $\mathrm{N}$-terminal sequence extension that associates it with intracellular membranes such as the Golgi and endosomes. It appears to interact predominantly with RhoB and RhoG. As their name implies, RhoGDIs function to inhibit nucleotide dissociation from Rho GTPases. They prevent GEF-mediated exchange and inhibit GAP activity. For RhoGDI1 and 2, a critical function is their ability to extract Rho GTPases from membranes, where normally the GTPases interact with downstream effectors. Recent studies have revealed that RhoGDI1 promotes the stability of Rho GTPases and protects them from degradation ${ }^{42,43}$. Interestingly, RhoGDI1 is expressed in cells at a level that is approximately equal to the sum of the major Rho family members ${ }^{41}$. This implies that it acts as a limited reservoir for the Rho proteins, with individual members competing for binding. 
Overexpression of one Rho family member was found to displace other Rho proteins from RhoGDI1, leading to their degradation and inactivation ${ }^{43}$. In some situations, however, the competitive displacement of one Rho protein by increased binding of another activates the displaced GTPase ${ }^{44}$. Phosphorylation of RhoA on serine 188 by PKA or PKG increases the affinity of RhoA binding to RhoGDI1 and in vascular smooth muscle this was shown to displace bound Rac1, which was translocated to the membrane and activated by the GEF, Vav $3^{44}$. This result suggests that the competition for binding to RhoGDI by Rho proteins may not be as coarse a regulatory mechanism as originally envisaged, but may allow modifications in binding affinity of one Rho protein to modulate the release and stability and/or activation of others.

The competitive binding to RhoGDI provides a mechanism for crosstalk between Rho proteins at the level of protein stability and degradation. The literature suggests that crosstalk may also occur through as yet uncharacterized transcriptional pathways. There is evidence that this may occur within the Rho subfamily, which includes RhoA, B and $C^{42,45}$. These closely related proteins share certain characteristics, such as the ability to induce stress fibers, but they also have unique functions. RhoA, for example, is required for mitosis in fibroblasts and cannot be substituted by $\mathrm{RhoB}$ or $\mathrm{RhoC}^{46}$. In some situations, these family members can exhibit opposite functions, as illustrated by the effects of RhoA and RhoC on cell migration and invasion ${ }^{45}$. Similarly, RhoB has the properties of a tumor suppressor, being pro-apoptotic, whereas RhoA and $\mathrm{C}$ have characteristics closer to being oncogenes ${ }^{47}$. Depletion of either RhoA or RhoC expression leads to a marked increase in RhoB levels. With the individual depletion of either RhoA or RhoC, little effect on the transcription of RhoB was observed, but simultaneous depletion of both proteins greatly increased RhoB mRNA levels ${ }^{42}$, indicating a level of regulation at the transcriptional level that will be interesting to explore further.

Studying the mechanism by which RhoB expression is influenced by RhoA or RhoC levels, it was discovered that the half life of RhoB, which is normally short-lived, is greatly increased by the knockdown of these other family members. RhoGDI1 is the crucial component mediating the interaction between these family members ${ }^{42}$. Overexpression of RhoGDI1 increased the half-life of RhoB, whereas its depletion inhibited the effect of RhoA on RhoB expression, leading to the conclusion that RhoGDI stabilizes RhoB against degradation ${ }^{42}$. Whereas this study provided evidence that the crosstalk did not involve RhoA activation ${ }^{42}$, a different group observed that inhibiting Rho activity with the $\mathrm{C} 3$ toxin also increased RhoB expression, implying that active Rho proteins are required for some part of this interaction ${ }^{45}$.

\section{Crosstalk via regulation of the same downstream signaling (target or effector)}

In this type of crosstalk, two (or more) Rho proteins share the same effector or molecular target (Figure 1).

\section{Regulation of the same effector}

Rac and Cdc42 have in common many effectors, including PAK1-3, Ncf1/2 and IQGAP5. RhoA and Rac have also been shown to share some downstream effectors. Early work on the kinase PRK2 showed that it interacts with both Rac and RhoA, in both cases leading to stimulation of its kinase activity ${ }^{48}$. More recent studies, however, observed that PRK2 acts mainly downstream of RhoA in vivo to regulate apical junctions ${ }^{49}$, suggesting that the crosstalk occurring through PRK2 may only happen under certain circumstances in vivo. The mDia formins are regulated by multiple Rho proteins. RhoA, B, and $\mathrm{C}^{50}$ and Rif ${ }^{51}$ can 
activate mDia1. Whereas, in addition to RhoA, $\mathrm{Rif}^{52}$, Rac and $\mathrm{Cdc} 42^{50}$ activate $\mathrm{mDia} 2$. Surprisingly this occurs through interaction with the same domain ${ }^{50}$, despite their weak similarity.

\section{Regulation of myosin light chain phosphorylation}

In nonmuscle cells, myosin II-generated tension is regulated by phosphorylation of myosin light chains (MLC). Several kinases have been identified that promote this phosphorylation, either directly or indirectly by inhibiting the MLC phosphatase. These include ROCK1 and 2 , which are activated by RhoA, and MRCK, which is activated by $\mathrm{Cdc} 42^{53}$. The Rac and Cdc42 effector PAK, on the other hand, can have opposite effects on MLC phosphorylation depending on the cell type analyzed. In Hela and BHK cells, PAK inhibits MLC phosphorylation and cell contractility by phosphorylating and inhibiting the myosin light chain kinase ${ }^{54}$, whereas in $3 \mathrm{~T} 3$ and endothelial cells PAK increases MLC phosphorylation ${ }^{55,56}$, although the mechanism has not been determined. More recently, it was shown that Rac1 inhibits MLC phosphorylation in melanoma cells, however, in this study WAVE2 mediated this effect rather than PAK1, 2 or $3^{11}$. Together, these results suggest that Rac and RhoA may act synergistically on MLC phosphorylation and cellular contractility in some cells but antagonistically in others.

\section{Regulation of cofilin}

The ADF/cofilin family of actin binding proteins promote actin filament disassembly ${ }^{57}$. In 1998, two groups independently reported that Rac regulates actin dynamics through, in part, LIMK1-dependent phosphorylation and inhibition of cofilin. Working in vivo and in vitro, both groups showed that Rac activates LIM-kinase 1, which in turn phosphorylates cofilin $^{58,59}$ on serine 3 and decreases its binding to actin. It was subsequently shown that both Rac and Cdc42 regulate LIMK activity through PAK1 ${ }^{60}$ and that RhoA can also regulate LIMK $2^{61}$ and LIMK $1^{62}$ via ROCK-dependent phosphorylation, leading to phosphorylation and functional inhibition of cofilin. These findings demonstrate that RhoA, Rac and Cdc42 act synergistically on cofilin and control actin filament stability through LIMK-dependent phosphorylation of cofilin. This crosstalk between RhoA, Rac and Cdc42 plays a central role during neuronal growth ${ }^{57}$.

\section{Rho protein coordination in cell adhesion and motility Integrin-based adhesion: switching from Rac1 to RhoA}

Since the discovery that Rho proteins control cell adhesion to extracellular matrix $(\mathrm{ECM})^{63,64}$, the relationship between individual Rho proteins and the assembly of adhesions has been extensively investigated. As cells adhere to the ECM, RhoA and Rac play distinct and opposing roles. Rac promotes formation of nascent adhesions and couples these small adhesions $(<0.5 \mu \mathrm{m})$ with actin-based protrusion near the cell periphery, whereas RhoA-dependent contractility produces changes in adhesion composition, leading to formation of larger $(>1 \mu \mathrm{m})$ more mature focal adhesions ${ }^{65,66}$ (figure 3 ). Consistent with these respective roles, initial adhesion and spreading are associated with transient RhoA inhibition $^{67}$ and Rac activation ${ }^{68}$, followed later by gradual RhoA activation and Rac inhibition. The balance between RhoA and Rac seems to control the fate of adhesions and much effort has been directed recently to identifying the GEFs and GAPs that orchestrate this switch.

Different pathways have been proposed for the regulation of Rac during the early phase of adhesion and various GEFs and GAPs suggested to play a role, including $\beta$-PIX ${ }^{12,} 69, \alpha$ $\mathrm{PIX}^{70}$ and $\mathrm{CdGAP}^{71}$. Since the identification of the DOCK180/ELMO pathway ${ }^{27}$, RhoG has been suggested to activate Rac downstream of integrin engagement ${ }^{72}$. It was recently 
shown, however, that RhoG depletion does not affect adhesion-dependent Rac activation ${ }^{73}$. Concomitant with Rac activation, we and others showed that RhoA inhibition is mediated by p190RhoGAP ${ }^{74-76}$. Interestingly, Rac regulates directly ${ }^{14}$, and indirectly through ROS generation ${ }^{15}$, p190RhoGAP activity and localization. This suggests that Rac may contribute to inhibit RhoA locally by activating and recruiting p190RhoGAP to nascent adhesions. More recently, it was found that PKA is activated in an adhesion-dependent manner at the leading edge of migrating epithelial cells and phosphorylates RhoA on Ser 188, increasing its affinity for $\mathrm{GDI}^{77}$. The authors showed that the resulting increased association of RhoA with GDI inhibits RhoA. As a consequence of the competitive binding on GDI that occurs between Rho proteins (see above discussion), one could anticipate that PKA-dependent RhoA phosphorylation may also induce Rac1 dissociation from $\mathrm{GDI}^{43}$ and its activation ${ }^{78}$. Consistent with this idea, integrin-dependent adhesion was discovered to promote Rac1 dissociation from GDI, leading to interaction with its effectors ${ }^{79}$.

Subsequent to this first phase controlled by Rac1, Rac1 activity decreases while RhoA becomes activated, promoting the growth and maturation of the nascent adhesions into focal adhesions. Different GEFs have been suggested to play a role in this process including $\mathrm{LARG}^{80}, \mathrm{p} 115 \mathrm{RhoGEF}^{80}, \mathrm{p} 190 \mathrm{RhoGEF}^{75}$ and GEF-H1 ${ }^{12}$. Interestingly, the Rac/Cdc42 effector PAK regulates the activity of $\mathrm{p} 115 \mathrm{RhoGEF}^{17}$ and GEF-H1 ${ }^{21,22}$, suggesting that Rac may potentially prevent RhoA activation and adhesion maturation during early spreading by inhibiting these two GEFs. Recent evidence suggests that, as adhesion progresses, RhoA activation may control the local inhibition of Rac. A mass spectrometry approach revealed how the adhesion proteome changes upon myosin inhibition ${ }^{12}$. It was found that $\beta$-PIX recruitment to adhesions is negatively regulated by contractility. These results suggest that RhoA-dependent myosin II activation triggers $\beta$-PIX dissociation from the adhesion and inhibits Rac1 locally ${ }^{12}$. Certainly, the development of mechanical tension contributes in several ways to the maturation of adhesions. Recent work has demonstrated that tension applied to integrins activates both GEF-H1 and LARG, although via different pathways, contributing to increased RhoA activity ${ }^{81}$.

\section{Cell migration}

Cell migration can be divided into distinct steps: protrusion of the leading edge, formation of new adhesions, cell body contraction and rear detachment ${ }^{82}$. Since actin cytoskeleton dynamics constitute the driving force during these steps, it is not surprising that Rho proteins have been implicated in regulating cell migration. Numerous studies have demonstrated that the prototypical members of the Rho family, RhoA, Rac1 and Cdc42, have specific roles during cell migration ${ }^{1}$. Recently, the development of fluorescent resonance energy transfer (FRET) -based biosensors that allow the visualization of spatiotemporal Rho signaling has demonstrated that RhoA, Rac1 and Cdc42 signal within distinct and specific zones during cell migration ${ }^{83-86}$. This confirms and spectacularly illustrates the idea that Rho proteins cooperate during migration ${ }^{87}$, suggesting that coordination and crosstalk between the Rho family members are essential to achieve efficient movement (Figure 3).

Rac1 regulates actin polymerization in the lamellipodial protrusion and promotes the formation of nascent adhesion near the cell periphery ${ }^{88}$. Using live-cell imaging of Rac biosensors in migrating neutrophils, it was found, as expected, that Rac1 is active in the extending leading edge ${ }^{85}$ of the cell. However, the authors also observed Rac1 activity at the rear where there is no actin-based protrusion ${ }^{85}$, indicating that Rac1 may play a different role in these two areas. Nevertheless, photoactivation of a caged constitutively active Rac1 construct in any region of a fibroblast appears sufficient to induce lamellipodial extension ${ }^{89}$.

Cdc42 has been shown to regulate the polarity of cell migration through different mechanisms. Cdc42 was shown to limit Rac1 activity at the front of migrating cells through 
PAK-mediated regulation of the Rac-specific GEF $\beta-P i x{ }^{90}$. The same group also showed that $\mathrm{Cdc} 42$ regulates microtubule polarity during directed migration by activating the atypical PKC in the Par6/aPKC complex at the leading edge ${ }^{91,92}$. Interestingly, the Rac GEF Tiam1 associates with the Par complex and is necessary for polarity establishment during keratinocytes migration ${ }^{93}$, suggesting that $\mathrm{Cdc} 42$ may control cellular polarity mainly through defining Rac1 activation area.

RhoA seems to play a role in every cellular compartment during cell migration ${ }^{1}$. RhoA is most commonly considered in the context of generating the contractile force that promotes cell body retraction at the rear through ROCK-mediated MLC phosphorylation ${ }^{94}$. However, it can also contribute to the extension of the leading lamella, as shown first with colon carcinoma cells migrating on laminin ${ }^{95}$. FRET-based biosensor imaging revealed that RhoA is active at the leading edge ${ }^{83}$. Through its interaction with the formin, mDia, RhoA drives actin polymerization ${ }^{96}$, but $\mathrm{mDia}$ has also been shown to activate $\mathrm{Rac} 1^{9}$, providing another example of RhoA crosstalk with Rac1 in the coordination of migration. RhoA also contributes to maintain cellular polarity by limiting inappropriate lateral protrusion ${ }^{8,45}$. Most likely this occurs through ROCK-mediated Rac1 inhibition and local inhibition of the Rac GEF $\beta$-PIX ${ }^{12,13}$ and/or activation of the Rac GAPs Arhgap22 ${ }^{11}$ or FilGAP ${ }^{10}$. Recently, it was shown that the different ROCK subtypes mediate different functions, with a role for ROCK1 in promoting cell body retraction, and ROCK2 inhibiting Rac1 and preventing protrusion ${ }^{45}$. In order for protrusion to occur at the front of cells, it is presumably important that the inhibitory function of ROCK2 be suppressed. Consistent with this idea, ROCK2 is inhibited by adhesion-induced tyrosine phosphorylation ${ }^{97}$, which can be anticipated to occur as the front of an advancing cell engages the ECM. Thus, RhoA plays distinct roles at the front and at the back of migrating cells, most likely through interacting with different sets of effectors at the different sites.

Working with melanoma cells, new crosstalk mechanisms were identified between RhoA and Rac $1^{11}$. Depending on the environmental conditions, individual tumor cells have two modalities of movement: a mesenchymal mode characterized by an elongated morphology and an amoeboid mode associated with high ROCK activity ${ }^{98}$. The authors showed that the mesenchymal mode is controlled by Rac1, which signals to WAVE2 to inhibit contractility and the amoeboid mode of migration ${ }^{11}$. Conversely, during amoeboid movement, ROCK inhibits Rac1 by stimulating the Rac GAP, ArhGAP22.

\section{Concluding remarks}

Although a considerable amount has been learned about the crosstalk between different Rho GTPases, much of this understanding is at the upstream level involving GEFs and GAPs. In contrast, less is known about the crosstalk that occurs downstream and that involves the interactions of the various signaling pathways initiated by the Rho GTPases. It is anticipated that there is much to be uncovered about crosstalk at the level of Rho protein effectors. Additionally, we expect that there is more to learn about how Rho GTPases can affect each other's expression, either at the transcriptional level or by influencing protein stability and degradation. When considering a complex behavior such as cell migration, it is striking that there are many levels of crosstalk occurring often simultaneously in different regions of the cell, such as at the leading edge, within the different types of adhesions, at the cell margins and in the cell rear. Many of these sites of crosstalk involve distinct protein complexes. The role of scaffold proteins in the assembly of these complexes and how they may contribute to the regulation of Rho protein crosstalk is poorly understood but promises to be a rich area of future investigation. 
The crosstalk between Rho family members described in this brief review represents just a small corner of the interactions between members of the Ras superfamily. Indeed, the first paper describing an interaction between Rac and Rho, also revealed that Ras itself can signal to $\mathrm{Rac}^{2}$. Numerous additional relationships have been discovered in which Ras family GTPases including Rab, Arf and Rap GTPases, affect the activities and signaling of Rho GTPases and vice versa. This interdependence helps coordinate a vast array of cellular processes, including migration, adhesion, membrane traffic and cell divison. The proliferation of crosstalk mechanisms within the Ras superfamily parallels the expansion of metazoans and has been accompanied by the emergence of new family members. Our understanding of this network of interactions will continue to increase, being driven by technological advances in proteomics, live-cell imaging and systems biology.

\section{Acknowledgments}

The authors apologize to the many groups whose work they could not include due to space limitations. CG thanks the European Union Seventh Framework Programme (FP7/2007-2013) for a Marie Curie Outgoing International fellowship (254747), RGM gratefully acknowledges support from the Simmons Scholars Program, KB thanks the Kenan Foundation for support and support from grants from the NIH (GM029860 and HL080166).

\section{References}

1. Jaffe AB, Hall A. Rho GTPases: biochemistry and biology. Annu Rev Cell Dev Biol. 2005; 21:247269. [PubMed: 16212495]

2. Ridley AJ, et al. The small GTP-binding protein rac regulates growth factor-induced membrane ruffling. Cell. 1992; 70:401-410. [PubMed: 1643658]

3. Rossman KL, et al. GEF means go: turning on RHO GTPases with guanine nucleotide-exchange factors. Nat Rev Mol Cell Biol. 2005; 6:167-180. [PubMed: 15688002]

4. Bos JL, et al. GEFs and GAPs: critical elements in the control of small G proteins. Cell. 2007; 129:865-877. [PubMed: 17540168]

5. Bustelo XR, et al. GTP-binding proteins of the Rho/Rac family: regulation, effectors and functions in vivo. Bioessays. 2007; 29:356-370. [PubMed: 17373658]

6. Garcia-Mata R, et al. The 'invisible hand': regulation of RHO GTPases by RHOGDIs. Nat Rev Mol Cell Biol. 2011; 12:493-504. [PubMed: 21779026]

7. Sander EE, et al. Rac downregulates Rho activity: reciprocal balance between both GTPases determines cellular morphology and migratory behavior. J Cell Biol. 1999; 147:1009-1022. [PubMed: 10579721]

8. Worthylake RA, Burridge K. RhoA and ROCK promote migration by limiting membrane protrusions. J Biol Chem. 2003; 278:13578-13584. [PubMed: 12574166]

9. Tsuji T, et al. ROCK and mDia1 antagonize in Rho-dependent Rac activation in Swiss 3T3 fibroblasts. J Cell Biol. 2002; 157:819-830. [PubMed: 12021256]

10. Ohta Y, et al. FilGAP, a Rho- and ROCK-regulated GAP for Rac binds filamin A to control actin remodelling. Nat Cell Biol. 2006; 8:803-814. [PubMed: 16862148]

11. Sanz-Moreno V, et al. Rac activation and inactivation control plasticity of tumor cell movement. Cell. 2008; 135:510-523. [PubMed: 18984162]

12. Kuo JC, et al. Analysis of the myosin-II-responsive focal adhesion proteome reveals a role for beta-Pix in negative regulation of focal adhesion maturation. Nat Cell Biol. 2011; 13:383-393. [PubMed: 21423176]

13. Vicente-Manzanares M, et al. Myosin IIA/IIB restrict adhesive and protrusive signaling to generate front-back polarity in migrating cells. J Cell Biol. 2011; 193:381-396. [PubMed: 21482721]

14. Bustos RI, et al. Coordination of Rho and Rac GTPase function via p190B RhoGAP. Curr Biol. 2008; 18:1606-1611. [PubMed: 18948007]

15. Nimnual AS, et al. Redox-dependent downregulation of Rho by Rac. Nat Cell Biol. 2003; 5:236241. [PubMed: 12598902] 
16. Aghajanian A, et al. Direct activation of RhoA by reactive oxygen species requires a redoxsensitive motif. PLoS One. 2009; 4:e8045. [PubMed: 19956681]

17. Rosenfeldt H, et al. Rac inhibits thrombin-induced Rho activation: evidence of a Pak-dependent GTPase crosstalk. J Mol Signal. 2006; 1:8. [PubMed: 17224083]

18. Alberts AS, et al. PAK1 negatively regulates the activity of the Rho exchange factor NET1. J Biol Chem. 2005; 280:12152-12161. [PubMed: 15684429]

19. Barac A, et al. Direct interaction of p21-activated kinase 4 with PDZ-RhoGEF, a G protein-linked Rho guanine exchange factor. J Biol Chem. 2004; 279:6182-6189. [PubMed: 14625312]

20. Arias-Romero LE, Chernoff J. A tale of two Paks. Biol Cell. 2008; 100:97-108. [PubMed: 18199048]

21. Zenke FT, et al. p21-activated kinase 1 phosphorylates and regulates 14-3-3 binding to GEF-H1, a microtubule-localized Rho exchange factor. J Biol Chem. 2004; 279:18392-18400. [PubMed: 14970201]

22. Callow MG, et al. PAK4 mediates morphological changes through the regulation of GEF-H1. J Cell Sci. 2005; 118:1861-1872. [PubMed: 15827085]

23. Cheng L, et al. Pleckstrin homology domain-mediated activation of the rho-specific guanine nucleotide exchange factor Dbs by Rac1. J Biol Chem. 2004; 279:12786-12793. [PubMed: 14701795]

24. Horii Y, et al. A novel oncogene, ost, encodes a guanine nucleotide exchange factor that potentially links Rho and Rac signaling pathways. EMBO J. 1994; 13:4776-4786. [PubMed: 7957046]

25. Liu Z, et al. The rho-specific guanine nucleotide exchange factor Dbs regulates breast cancer cell migration. J Biol Chem. 2009; 284:15771-15780. [PubMed: 19366686]

26. Gauthier-Rouviere C, et al. RhoG GTPase controls a pathway that independently activates Rac1 and Cdc42Hs. Mol Biol Cell. 1998; 9:1379-1394. [PubMed: 9614181]

27. Katoh H, Negishi M. RhoG activates Rac1 by direct interaction with the Dock180-binding protein Elmo. Nature. 2003; 424:461-464. [PubMed: 12879077]

28. Brugnera E, et al. Unconventional Rac-GEF activity is mediated through the Dock180-ELMO complex. Nat Cell Biol. 2002; 4:574-582. [PubMed: 12134158]

29. Gumienny TL, et al. CED-12/ELMO, a novel member of the CrkII/Dock180/Rac pathway, is required for phagocytosis and cell migration. Cell. 2001; 107:27-41. [PubMed: 11595183]

30. Blangy A, et al. TrioGEF1 controls Rac- and Cdc42-dependent cell structures through the direct activation of rhoG. J Cell Sci. 2000; 113(Pt 4):729-739. [PubMed: 10652265]

31. Samson T, et al. Endogenous RhoG is rapidly activated after epidermal growth factor stimulation through multiple guanine-nucleotide exchange factors. Mol Biol Cell. 2010; 21:1629-1642. [PubMed: 20237158]

32. Wennerberg K, et al. RhoG signals in parallel with Rac1 and Cdc42. J Biol Chem. 2002; 277:47810-47817. [PubMed: 12376551]

33. Prieto-Sanchez RM, Bustelo XR. Structural basis for the signaling specificity of RhoG and Rac1 GTPases. J Biol Chem. 2003; 278:37916-37925. [PubMed: 12805377]

34. Chardin P. Function and regulation of Rnd proteins. Nat Rev Mol Cell Biol. 2006; 7:54-62. [PubMed: 16493413]

35. Wennerberg K, et al. Rnd proteins function as RhoA antagonists by activating p190 RhoGAP. Curr Biol. 2003; 13:1106-1115. [PubMed: 12842009]

36. Riento K, et al. RhoE binds to ROCK 1 and inhibits downstream signaling. Molecular \& Cellular Biology. 2003; 23:4219-4229. [PubMed: 12773565]

37. Riento K, et al. RhoE function is regulated by ROCK I-mediated phosphorylation. Embo J. 2005; 24:1170-1180. [PubMed: 15775972]

38. Bateman J, Van Vactor D. The Trio family of guanine-nucleotide-exchange factors: regulators of axon guidance. J Cell Sci. 2001; 114:1973-1980. [PubMed: 11493634]

39. Chuang TH, et al. Abr and Bcr are multifunctional regulators of the Rho GTP-binding protein family. Proc Natl Acad Sci U S A. 1995; 92:10282-10286. [PubMed: 7479768] 
40. Vaughan EM, et al. Control of local Rho GTPase crosstalk by Abr. Curr Biol. 2011; 21:270-277. [PubMed: 21295482]

41. Michaelson D, et al. Differential localization of Rho GTPases in live cells: regulation by hypervariable regions and RhoGDI binding. Journal of Cell Biology. 2001; 152:111-126. [PubMed: 11149925]

42. Ho TT, et al. RhoA-GDP regulates RhoB protein stability. Potential involvement of RhoGDIalpha. J Biol Chem. 2008; 283:21588-21598. [PubMed: 18524772]

43. Boulter E, et al. Regulation of Rho GTPase crosstalk, degradation and activity by RhoGDI1. Nat Cell Biol. 2010; 12:477-483. [PubMed: 20400958]

44. Rolli-Derkinderen M, et al. RhoA phosphorylation induces Rac1 release from guanine dissociation inhibitor alpha and stimulation of vascular smooth muscle cell migration. Mol Cell Biol. 2010; 30:4786-4796. [PubMed: 20696841]

45. Vega FM, et al. RhoA and RhoC have distinct roles in migration and invasion by acting through different targets. J Cell Biol. 2011; 193:655-665. [PubMed: 21576392]

46. Melendez J, et al. RhoA GTPase is dispensable for actomyosin regulation but is essential for mitosis in primary mouse embryonic fibroblasts. J Biol Chem. 2011; 286:15132-15137. [PubMed: 21454503]

47. Wheeler AP, Ridley AJ. Why three Rho proteins? RhoA, RhoB, RhoC, and cell motility. Exp Cell Res. 2004; 301:43-49. [PubMed: 15501444]

48. Vincent S, Settleman J. The PRK2 kinase is a potential effector target of both Rho and Rac GTPases and regulates actin cytoskeletal organization. Mol Cell Biol. 1997; 17:2247-2256. [PubMed: 9121475]

49. Wallace SW, et al. The Rho target PRK2 regulates apical junction formation in human bronchial epithelial cells. Mol Cell Biol. 2011; 31:81-91. [PubMed: 20974804]

50. Lammers M, et al. Specificity of interactions between mDia isoforms and Rho proteins. J Biol Chem. 2008; 283:35236-35246. [PubMed: 18829452]

51. Fan L, et al. The small GTPase Rif is an alternative trigger for the formation of actin stress fibers in epithelial cells. J Cell Sci. 2010; 123:1247-1252. [PubMed: 20233848]

52. Pellegrin S, Mellor H. The Rho family GTPase Rif induces filopodia through mDia2. Curr Biol. 2005; 15:129-133. [PubMed: 15668168]

53. Vicente-Manzanares M, et al. Non-muscle myosin II takes centre stage in cell adhesion and migration. Nat Rev Mol Cell Biol. 2009; 10:778-790. [PubMed: 19851336]

54. Sanders LC, et al. Inhibition of myosin light chain kinase by p21-activated kinase. Science. 1999; 283:2083-2085. [PubMed: 10092231]

55. Sells MA, et al. p21-activated kinase 1 (Pak1) regulates cell motility in mammalian fibroblasts. J Cell Biol. 1999; 145:837-849. [PubMed: 10330410]

56. Kiosses WB, et al. A role for p21-activated kinase in endothelial cell migration. J Cell Biol. 1999; 147:831-844. [PubMed: 10562284]

57. Kuhn TB, et al. Regulating actin dynamics in neuronal growth cones by ADF/cofilin and rho family GTPases. J Neurobiol. 2000; 44:126-144. [PubMed: 10934317]

58. Yang N, et al. Cofilin phosphorylation by LIM-kinase 1 and its role in Rac-mediated actin reorganization. Nature. 1998; 393:809-812. [PubMed: 9655398]

59. Arber S, et al. Regulation of actin dynamics through phosphorylation of cofilin by LIM-kinase. Nature. 1998; 393:805-809. [PubMed: 9655397]

60. Edwards DC, et al. Activation of LIM-kinase by Pak1 couples Rac/Cdc42 GTPase signalling to actin cytoskeletal dynamics. Nat Cell Biol. 1999; 1:253-259. [PubMed: 10559936]

61. Sumi T, et al. Cofilin phosphorylation and actin cytoskeletal dynamics regulated by rho- and Cdc42-activated LIM-kinase 2. J Cell Biol. 1999; 147:1519-1532. [PubMed: 10613909]

62. Ohashi K, et al. Rho-associated kinase ROCK activates LIM-kinase 1 by phosphorylation at threonine 508 within the activation loop. J Biol Chem. 2000; 275:3577-3582. [PubMed: 10652353]

63. Paterson HF, et al. Microinjection of recombinant $\mathrm{p} 21$ rho induces rapid changes in cell morphology. J Cell Biol. 1990; 111:1001-1007. [PubMed: 2118140] 
64. Ridley AJ, Hall A. The small GTP-binding protein rho regulates the assembly of focal adhesions and actin stress fibers in response to growth factors. Cell. 1992; 70:389-399. [PubMed: 1643657]

65. Chrzanowska-Wodnicka M, Burridge K. Rho-stimulated contractility drives the formation of stress fibers and focal adhesions. J Cell Biol. 1996; 133:1403-1415. [PubMed: 8682874]

66. Choi CK, et al. Actin and alpha-actinin orchestrate the assembly and maturation of nascent adhesions in a myosin II motor-independent manner. Nat Cell Biol. 2008

67. Ren XD, et al. Regulation of the small GTP-binding protein Rho by cell adhesion and the cytoskeleton. EMBO Journal. 1999; 18:578-585. [PubMed: 9927417]

68. Price LS, et al. Activation of Rac and Cdc42 by integrins mediates cell spreading. Molecular Biology of the Cell. 1998; 9:1863-1871. [PubMed: 9658176]

69. Boulter E, et al. Regulation of cell-matrix adhesion dynamics and Rac-1 by integrin linked kinase. FASEB J. 2006; 20:1489-1491. [PubMed: 16723384]

70. Filipenko NR, et al. Integrin-linked kinase activity regulates Rac- and Cdc42-mediated actin cytoskeleton reorganization via alpha-PIX. Oncogene. 2005; 24:5837-5849. [PubMed: 15897874]

71. LaLonde DP, et al. CdGAP associates with actopaxin to regulate integrin-dependent changes in cell morphology and motility. Curr Biol. 2006; 16:1375-1385. [PubMed: 16860736]

72. Katoh H, et al. Activation of Rac1 by RhoG regulates cell migration. J Cell Sci. 2006; 119:56-65. [PubMed: 16339170]

73. Meller J, et al. Endogenous RhoG is dispensable for integrin-mediated cell spreading but contributes to Rac-independent migration. J Cell Sci. 2008; 121:1981-1989. [PubMed: 18505794]

74. Arthur WT, Burridge K. RhoA inactivation by p190RhoGAP regulates cell spreading and migration by promoting membrane protrusion and polarity. Mol Biol Cell. 2001; 12:2711-2720. [PubMed: 11553710]

75. Lim Y, et al. PyK2 and FAK connections to p190Rho guanine nucleotide exchange factor regulate RhoA activity, focal adhesion formation, and cell motility. J Cell Biol. 2008; 180:187-203. [PubMed: 18195107]

76. Peacock JG, et al. The Abl-related gene tyrosine kinase acts through p190RhoGAP to inhibit actomyosin contractility and regulate focal adhesion dynamics upon adhesion to fibronectin. Mol Biol Cell. 2007; 18:3860-3872. [PubMed: 17652459]

77. Tkachenko E, et al. Protein kinase A governs a RhoA-RhoGDI protrusion-retraction pacemaker in migrating cells. Nat Cell Biol. 13:661-668.

78. Rolli-Derkinderen M, et al. RhoA phosphorylation induces Rac1 release from guanine dissociation inhibitor alpha and stimulation of vascular smooth muscle cell migration. Mol Cell Biol. 30:47864796. [PubMed: 20696841]

79. Del Pozo MA, et al. Integrins regulate GTP-Rac localized effector interactions through dissociation of Rho-GDI. Nat Cell Biol. 2002; 4:232-239. [PubMed: 11862216]

80. Dubash AD, et al. A novel role for Lsc/p115 RhoGEF and LARG in regulating RhoA activity downstream of adhesion to fibronectin. J Cell Sci. 2007; 120:3989-3998. [PubMed: 17971419]

81. Guilluy C, et al. The Rho GEFs LARG and GEF-H1 regulate the mechanical response to force on integrins. Nat Cell Biol. 2011; 13:724-729.

82. Lauffenburger DA, Horwitz AF. Cell migration: a physically integrated molecular process. Cell. 1996; 84:359-369. [PubMed: 8608589]

83. Machacek M, et al. Coordination of Rho GTPase activities during cell protrusion. Nature. 2009; 461:99-103. [PubMed: 19693013]

84. Pertz O, et al. Spatiotemporal dynamics of RhoA activity in migrating cells. Nature. 2006; 440:1069-1072. [PubMed: 16547516]

85. Gardiner EM, et al. Spatial and temporal analysis of Rac activation during live neutrophil chemotaxis. Curr Biol. 2002; 12:2029-2034. [PubMed: 12477392]

86. Itoh RE, et al. Activation of rac and cdc 42 video imaged by fluorescent resonance energy transferbased single-molecule probes in the membrane of living cells. Mol Cell Biol. 2002; 22:6582-6591. [PubMed: 12192056]

87. Nobes CD, Hall A. Rho GTPases control polarity, protrusion, and adhesion during cell movement. Journal of Cell Biology. 1999; 144:1235-1244. [PubMed: 10087266] 
88. Gardel ML, et al. Mechanical integration of actin and adhesion dynamics in cell migration. Annu Rev Cell Dev Biol. 2010; 26:315-333. [PubMed: 19575647]

89. Wu YI, et al. A genetically encoded photoactivatable Rac controls the motility of living cells. Nature. 2009; 461:104-108. [PubMed: 19693014]

90. Cau J, Hall A. Cdc42 controls the polarity of the actin and microtubule cytoskeletons through two distinct signal transduction pathways. J Cell Sci. 2005; 118:2579-2587. [PubMed: 15928049]

91. Etienne-Manneville S, Hall A. Integrin-mediated activation of $\mathrm{Cdc} 42$ controls cell polarity in migrating astrocytes through PKCzeta. Cell. 2001; 106:489-498. [PubMed: 11525734]

92. Etienne-Manneville S, Hall A. Cdc42 regulates GSK-3beta and adenomatous polyposis coli to control cell polarity. Nature. 2003; 421:753-756. [PubMed: 12610628]

93. Pegtel DM, et al. The Par-Tiam1 complex controls persistent migration by stabilizing microtubuledependent front-rear polarity. Curr Biol. 2007; 17:1623-1634. [PubMed: 17825562]

94. Worthylake RA, et al. RhoA is required for monocyte tail retraction during transendothelial migration. J Cell Biol. 2001; 154:147-160. [PubMed: 11448997]

95. O'Connor KL, et al. RhoA function in lamellae formation and migration is regulated by the alpha6beta4 integrin and cAMP metabolism. Journal of Cell Biology. 2000; 148:253-258. [PubMed: 10648558]

96. Watanabe N, et al. p140mDia, a mammalian homolog of Drosophila diaphanous, is a target protein for Rho small GTPase and is a ligand for profilin. EMBO Journal. 1997; 16:3044-3056. [PubMed: 9214622]

97. Lee HH, Chang ZF. Regulation of RhoA-dependent ROCKII activation by Shp2. J Cell Biol. 2008; 181:999-1012. [PubMed: 18559669]

98. Friedl P, Wolf K. Plasticity of cell migration: a multiscale tuning model. J Cell Biol. 2010; 188:1119. [PubMed: 19951899] 


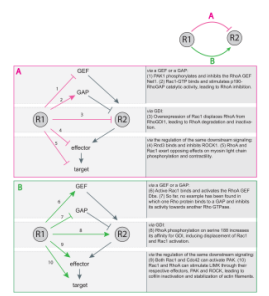

Figure 1. Modalities of regulation between two Rho proteins Diagram showing how two Rho proteins (R1 and R2) can negatively (A) or positively (B) regulate one another. An example is indicated for each type of modality (via a GEF or a GAP, via GDI, via the regulation of the same downstream signaling pathway). 


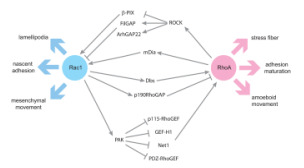

Figure 2. Crosstalk between RhoA and Rac1

Schematic diagram showing the crosstalk mechanisms between RhoA and Rac1. 


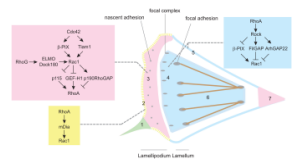

Figure 3. Rho protein crosstalk during cell migration

A diagram of a migrating fibroblast is shown depicting zones of Rho protein activation and crosstalk. 1. Cdc42 controls formation of exploratory filopodia. 2. RhoA activity has been detected at the leading edge of lamellipodia where it may contribute to actin polymerization, directly via mDia or indirectly through mDia activating Rac1. 3. Behind the narrow zone of high RhoA activity, a wider zone of high Rac1 activity has been described. This may arise downstream from integrin engagement. Alternatively, $\mathrm{Cdc} 42$ and RhoG may contribute to Rac1 activation. This, in turn, inhibits RhoA and promotes nascent adhesion formation associated with actin-based protrusion. 4. RhoA generates ROCK-mediated contractility and inhibits Rac1, leading to adhesion maturation. 5. RhoA prevents inappropriate lateral protrusion by inhibiting Rac1 through ROCK2. 6. RhoA promotes cell body retraction through ROCK1-mediated myosin II stimulation. 7. Rac1 activation at the tail has been described but its function in this area is unknown. 
Table 1

Rho GEFs and GAPs whose activity or localization are affected by another RhoGTPase

\begin{tabular}{|c|c|c|c|c|}
\hline Name & Type andspecificity & Regulated by & Mechanism & Effect \\
\hline p115-RhoGEF & GEF for RhoA, RhoB and Rhoc & Rac1 & $\begin{array}{l}\text { PAK1 phosphorylates p115-RhoGEF and inhibits p115- } \\
\text { RhoGEF-mediated RhoA activation }{ }^{15} \text {. }\end{array}$ & $(-)$ \\
\hline GEF-H1 & GEF for RhoA, RhoBand RhoC & Rac1 & $\begin{array}{l}\text { PAK1 and PAK4 phosphorylate GEF-H1 and affect its } \\
\text { localization }{ }^{19,20} \text {, leading to RhoA inhibition. }\end{array}$ & $(-)$ \\
\hline PDZ-RhoGEF & GEF for RhoA, RhoB and RhoC & Rac1 & $\begin{array}{l}\text { PAK4 phosphorylates PDZ-RhoGEF and inhibits LPA- } \\
\text { induced RhoA activation }{ }^{17} \text {. }\end{array}$ & $(-)$ \\
\hline Net1 & GEF for RhoA & Rac1 & PAK1 phosphorylates and inhibits Net $1^{16}$. & \\
\hline \multirow[t]{3}{*}{ p190-RhoGAP } & GAP for RhoA, RhoB and RhoC & Rac1 & -Rac1-GTP binds and activates p190-RhoGAP ${ }^{12}$. & $(-)$ \\
\hline & & & $\begin{array}{l}\text {-Rac1-mediated ROS production stimulates p190-RhoGAP } \\
\text { catalytic activity }{ }^{13} \text {. }\end{array}$ & $(-)$ \\
\hline & & Rnd1,3 & Rnd 1 and Rnd3 associate with and activate p190-RhoGAP 32 . & $(-)$ \\
\hline Dbs & GEF for RhoA and Cdc42 & Rac1 & Rac1-GTP binds and activates $\mathrm{Dbs}^{21}$. & $(+)$ \\
\hline ArhGAP22 & GAP for Rac 1 & RhoA & ROCK-mediated contractility activates ArhGAP $22^{10}$. & $(-)$ \\
\hline FilGAP & GAP for Rac1 & RhoA & ROCK phosphorylates and activates FilGAP ${ }^{9}$. & $(-)$ \\
\hline$\alpha$-PIX & GEF for Rac1 and $\mathrm{Cdc} 42$ & $\mathrm{Cdc} 42$ & $\begin{array}{l}\text { Cdc } 42 \text { activates PAK, which in turn associates with } \alpha \text {-PIX, } \\
\text { leading to local activation of } \operatorname{Rac} 1^{95} \text {. }\end{array}$ & $(+)$ \\
\hline \multirow[t]{2}{*}{$\beta$-PIX } & GEF for Rac1 and Cdc42 & RhoA & $\begin{array}{l}\text { ROCK-mediated contractility induces } \beta \text {-PIX dissociation } \\
\text { from integrin-based adhesion and local Rac } 1 \text { inhibition }{ }^{11} \text {. }\end{array}$ & $(-)$ \\
\hline & & $\mathrm{Cdc} 42$ & $\begin{array}{l}\text { Cdc } 42 \text { promotes PAK association with } \beta \text {-PIX and induces } \\
\text { local Rac } 1 \text { activation }{ }^{86} \text {. }\end{array}$ & $(+)$ \\
\hline Dock180 & GEF for Rac1 & RhoG & $\begin{array}{l}\text { ELMO associates with Dock } 180 \text { and induces its translocation } \\
\text { to the plasma membrane, leading to Rac } 1 \text { activation }{ }^{25} \text {. }\end{array}$ & $(+)$ \\
\hline
\end{tabular}

The effects (last column) are indicated as (-) for an inhibition and (+) for an activation. 